\title{
From deep-inelastic structure functions to two-photon dilepton production in proton-proton collisions
}

\author{
Antoni Szczurek ${ }^{* \dagger}$ \\ Institute of Nuclear Physics, Polish Academy of Sciences, Radzikowskiego 152, \\ PL-31-342 Kraków, Poland \\ E-mail: antoni.szczurek@ifj.edu.pl
}

Marta Łuszczak

Department of Theoretical Physics, University of Rzeszów, PL-35-959 Rzeszów, Poland

E-mail: luszczak@ur.edu.pl

\section{Wolfgang Schäfer}

Institute of Nuclear Physics, Polish Academy of Sciences, Radzikowskiego 152,

PL-31-342 Kraków, Poland

E-mail: wolfgang.schafereifj.edu.pl

\begin{abstract}
We compare two different approaches used for calculating cross sections for the two-photon $p p \rightarrow l^{+} l^{-} X$ process. In one of the approaches photon is treated as a collinear parton in the proton. In the second approach a recently proposed $k_{T}$-factorization method is used. In this presentation we discuss sensitivity of the results to the choice of structure function parametrization and experimental cuts in the $k_{T}$-factorization approach. We compare results of our calculations with recent experimental data for dilepton production and find that in most cases the contribution of the photon-photon mechanism is rather small. We discuss how to enhance the photon-photon contribution. We also compare our results to those of recent measurements of exclusive and semiexclusive $e^{+} e^{-}$pair production with certain experimental data by the CMS collaboration.
\end{abstract}

XXIV International Workshop on Deep-Inelastic Scattering and Related Subjects

11-15 April, 2016

DESY Hamburg, Germany

\footnotetext{
${ }^{*}$ Speaker.

${ }^{\dagger}$ The work has been supported by the Polish National Science Center grant DEC-2014/15/B/ST2/02528.
} 


\section{Introduction}

In this presentation we show our recent results published in [1] where we have considered $p p \rightarrow p p l^{+} l^{-}$(exclusive) and $p p \rightarrow l^{+} l^{-}$(semiexclusive, with proton dissociation) double photon fusion processes in the proposed somewhat earlier $k_{t}$-factorization approach [2]. In our presentation at DIS2016 we have focussed on the relation of the cross section for the charged lepton pair production with the dependence of the deep-inelastic structure functions $F_{2}$, that are the input for our approach, on $x$ and $Q^{2}$.

The main mechanisms of the dilepton production considered in [1] are shown in Fig.1. The considered mechanism has the same final state as the dominant Drell-Yan mechanism. For $k_{t^{-}}$ factorization approach to the Drell-Yan see [3, 4, 5, 6]. In our recent paper [1] we discussed in detail the photon-photon mechanism.
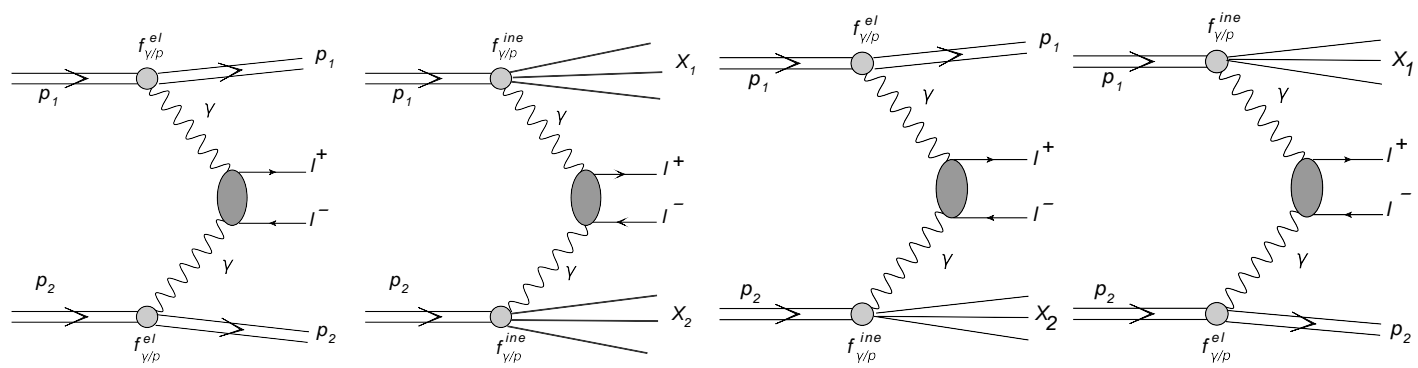

Figure 1:

Different mechanisms of two-photon production of dileptons included in [1].

\section{Basic formulae}

In collinear approximation the cross sections are calculated as:

$$
\frac{d \sigma^{\gamma_{i n}} \gamma_{\text {in }}}{d y_{1} d y_{2} d^{2} p_{t}}=\frac{1}{16 \pi^{2} \hat{s}^{2}} x_{1} \gamma_{i}\left(x_{1}, \mu^{2}\right) x_{2} \gamma_{j}\left(x_{2}, \mu^{2}\right) \overline{\left|\mathscr{M}_{\gamma \gamma \rightarrow l^{+} l^{-}}\right|^{2}},
$$

where $\mathrm{i}, \mathrm{j}=\mathrm{el}$,in and $f_{i}$ are photon PDFs. The elastic photon fluxes are calculated using the DreesZeppenfeld parametrization, where a simple parametrization of nucleon electromagnetic form factors was used.

In the $k_{t}$-factorization approach the differential cross section can be written as:

$$
\frac{d \sigma^{(i, j)}}{d y_{1} d y_{2} d^{2} p_{1} d^{2} p_{2}}=\int \frac{d^{2} q_{1}}{\pi q_{1}^{2}} \frac{d^{2} q_{2}}{\pi q_{2}^{2}} \mathscr{F}_{\gamma^{*} / A}^{(i)}\left(x_{1}, q_{1}\right) \mathscr{F}_{\gamma^{*} / B}^{(j)}\left(x_{2}, q_{2}\right) \frac{d \sigma^{*}\left(p_{1}, p_{2} ; q_{1}, q_{2}\right)}{d y_{1} d y_{2} d^{2} p_{1} d^{2} p_{2}},
$$

where i,j=el,ine and $\mathscr{F}_{k}$ are unintegrated fluxes of photons. As shown in [1] the unintegrated fluxes can be expressed in terms of the (deep-inelastic) structure functions $F_{2}\left(x, Q^{2}\right)$. 


\section{Numerical results}

In our studies in [1] we have used a few different parametrizations of the proton structure function $F_{2}$ taken from the literature:

- ALLM $[8,9]$. This parametrization gives a very good fit to $F_{2}$ in most of the measured region.

- FJLLM [10]. This parametrization explicitly includes the nucleon resonances and gives an excellent fit of the CLAS data.

- BDH [11]. This parametrization concentrates on the low- $x$, or high mass region. It features a Froissart-like behaviour at very small $x$.

- SY [12]. This paramerization of Suri and Yennie from the early 1970's does not include QCD-DGLAP evolution. It is still today often used as one of the defaults in the LPAIR event generator.

- SU [13]. A parametrization which concentrates to give a good description at rather small and intermediate $Q^{2}$ at not too small $x$.

We also show $F_{2}$ calculated from the CTEQ6L parametrization [14].

In Fig. 2 we show only two examples of the proton structure function $F_{2}\left(x, Q^{2}\right)$ obtained from the various parametrizations at $Q^{2}=2.5,4.5 \mathrm{GeV}^{2}$ as a function of Bjorken- $x$.

It is surprising that the old Suri-Yennie [12] fit, still gives a reasonable description of $F_{2}$ except of very small $x$. For explicit account of resonances we recommend to use the Fiore et al. [10], but care has to be taken to stay within the resonance region, as the quality of the fit beyond this region quickly deteriorates. The overall best description appears to be given by the ALLM $[8,9]$ fit.
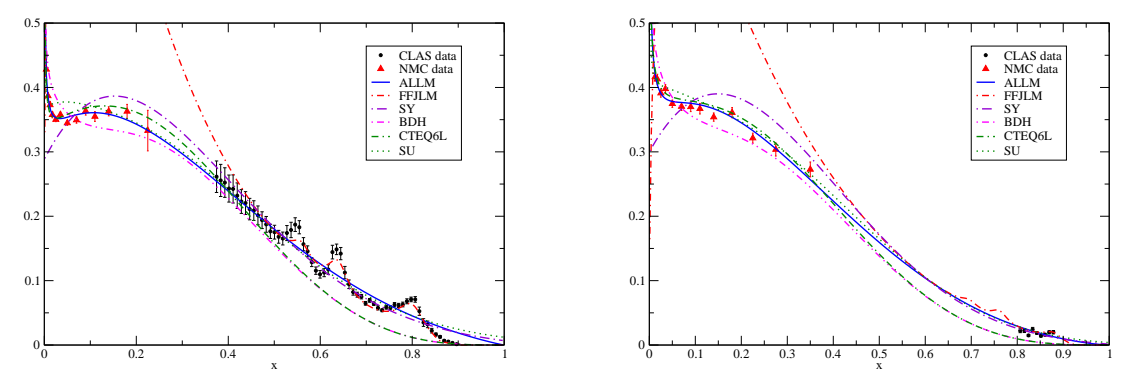

Figure 2: The proton structure function $F_{2}\left(x, Q^{2}\right)$ as a function of $x$ for $Q^{2}=2.5 \mathrm{GeV}^{2}$ (left), and $Q^{2}=$ $4.5 \mathrm{GeV}^{2}$ (right). Shown are results for different parmetrizations available in the literature.

In Ref.[1] we have compared our calculations with measured dilepton data [15, 16, 17, 18, 19]. Here we show only a few examples.

Most of the experiments for the dilepton production concentrate on determination of dilepton invariant mass distributions. In Fig. 3 we show invariant mass distributions of dilepton pairs produced in the photon-photon inelastic-inelastic mechanism for kinematical conditions relevant for 
different experiments. We show results obtained with the different parametrizations of the structure functions known from the literature. Surprisingly the different structure functions give quite different results. For completeness in some cases we also show the result obtained in the collinear approach with the MRST2004(QED) photon distribution [7] with (solid black line) and similar one when ignoring the initial input (long-dashed black line). The result obtained within the collinear approach with the MRST2004(QED) distribution is much above the results obtained within the $k_{t^{-}}$ factorization approach. In our opinion this is mainly related to the large input photon distribution at the initial scale $Q_{0}^{2}=2 \mathrm{GeV}^{2}$. If the input is discarded (long-dashed black line) the collinear result is similar to the results obtained within the $k_{T}$-factorization. The inelastic-inelastic contribution gives only a small fraction of the measured cross section for most experimental conditions (ATLAS,LHCb).
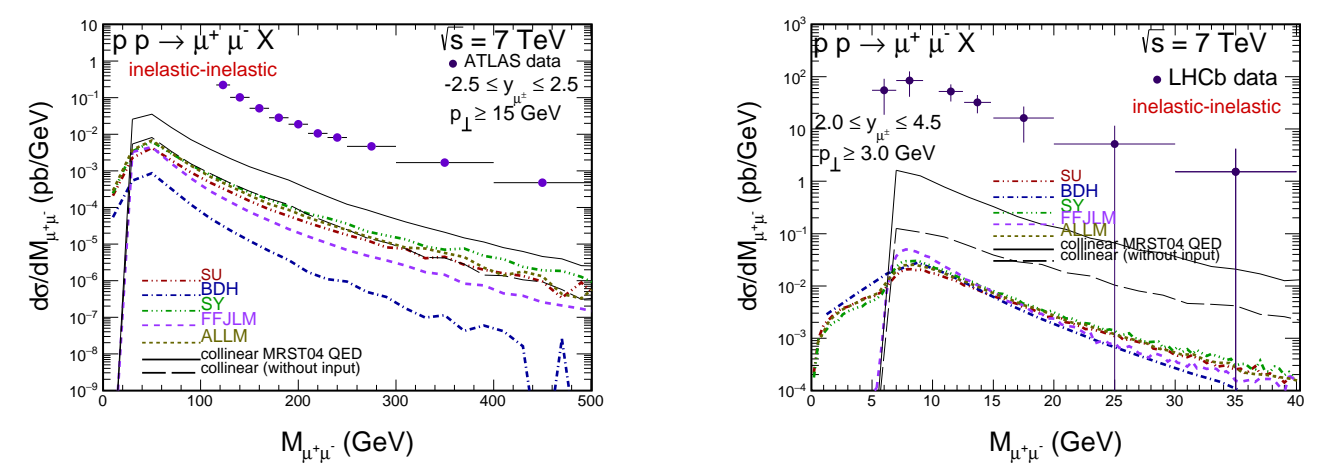

Figure 3: The inelastic-inelastic contribution to dilepton invariant mass distributions for ATLAS (left) and $\mathrm{LHCb}$ (right) experiments for different structure functions.

In Fig. 4 we show dilepton invariant mass distributions for elastic-inelastic and inelastic-elastic (added together) contributions. As for inelastic-inelastic contribution the results strongly depend on the parametrization of the structure functions used. The spread of results for different $F_{2}$ from the literature is now somewhat smaller than in the case of inelastic-inelastic contributions where the structure functions enter twice. As for the double inelastic case we also show a result for the collinear approach. The mixed components give similar contribution to the dilepton invariant mass distributions as the inelastic-inelastic one.

In most of the cases considered so far Drell-Yan processes dominate $[4,5,6]$. The twophoton processes are interesting by themselves. Can they be measured? In order to reduce the Drell-Yan contribution and relatively enhance the two-photon contribution one can impose an extra condition on lepton isolation. First trials have been done by the CMS collaboration [20]. In their analysis an extra lepton isolation cuts were imposed in order to eliminate the dominating Drell-Yan component. In Figs. 5,6,7 we show our results for two different (SY and ALLM) parametrizations of the structure functions for distributions in dimuon invariant mass, in transverse momentum of the pair and in relative azimuthal angle between $\mu^{+} \mu^{-}$. SY and ALLM parametrizations give almost the same contributions to all the distributions considered. In the first evaluation we have taken into account integrated luminosity of the experiment $\left(L=63.2 \mathrm{pb}^{-1}\right)$ as well as experimental acceptances given in Ref.[20]. Rather good agreement with the low statistics CMS experimental data is achieved without including any extra corrections due to absorption effects. It may mean that 

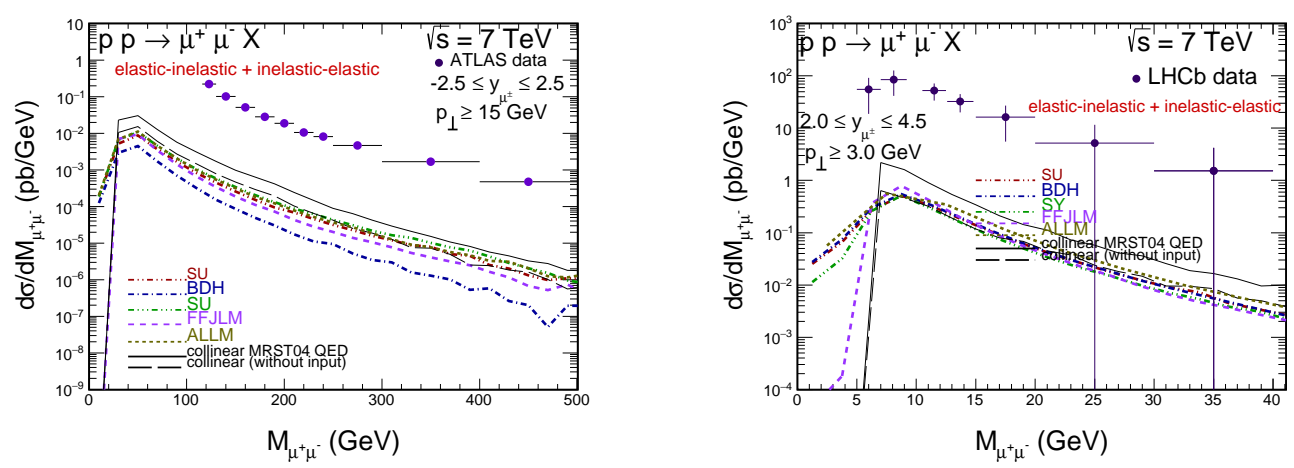

Figure 4: The (elastic-inelastic)+(inelastic-elastic) contribution to dilepton invariant mass distributions for ATLAS (left) and LHCb (right) experiments for different structure functions.

the absorption effects are small or alternatively that a contamination of the Drell-Yan contribution is still not completely removed. Both effects should be therefore studied in detail in a future.
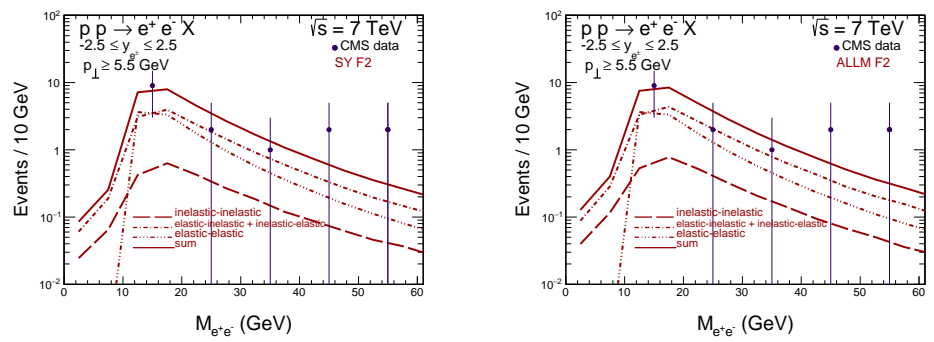

Figure 5: Number of events per invariant mass interval for the CMS experimental cuts for SY (left) and ALLM (right) structure functions. The experimental data points are from Ref.[20].
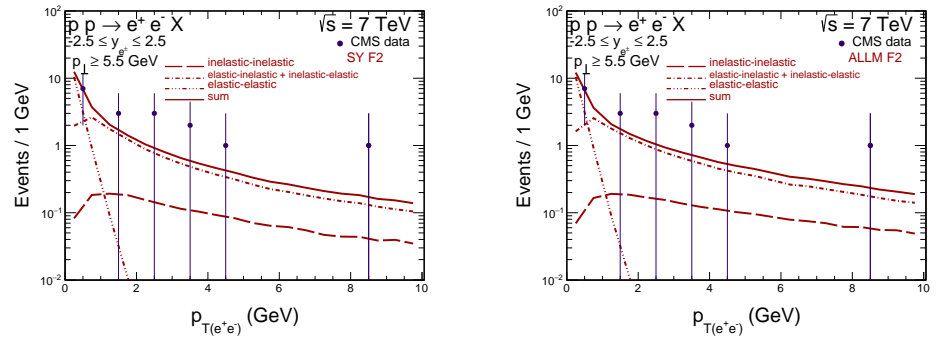

Figure 6: Number of events per pair transverse momentum interval for the CMS experimental cuts for SY (left) and ALLM (right) structure functions. The experimental data points are from Ref.[20]. 

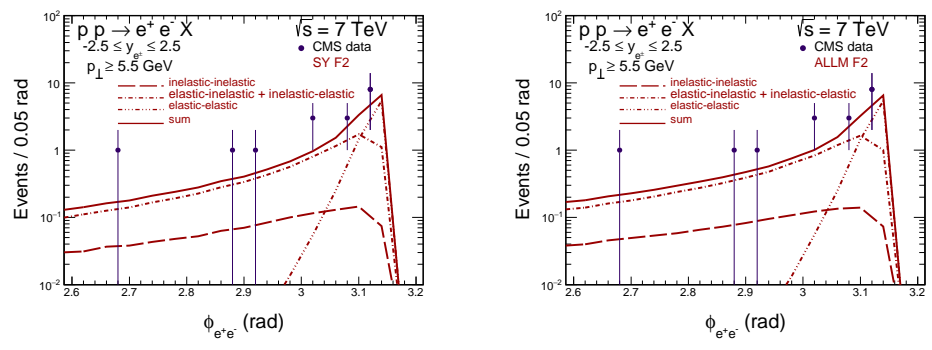

Figure 7: Number of events per pair relative azimuthal angle interval for the CMS experimental cut for SY (left) and ALLM (right) structure functions. The experimental data points are from Ref.[20].

\section{Conclusions}

We summarize our studies in [1] as follows:

- Two different approaches (collinear and $k_{t}$-factorization) for $\gamma \gamma \rightarrow l^{+} l^{-}$processes were discussed and compared.

- Strong dependence on the structure function input in the $k_{t}$-factorization approach were found.

- Semi-exclusive contributions with proton dissociation is large (this may be interesting lesson for other processes such as e.g. the $p p \rightarrow p p J / \psi$ reaction).

- Photon-photon contribution is rather small compared to Drell-Yan contribution but is important in precision calculations.

- Reasonable description of the CMS data with isolated electrons was achieved (recently also ATLAS obtained similar result).

- The regions of the arguments of the structure function $F_{2}$ important for the discussed $\gamma \gamma \rightarrow$ $l^{+} l^{-}$process was identified.

- So far only collinear approach was applied to $p p \rightarrow(\gamma \gamma) \rightarrow W^{+} W^{-} X Y$ processes which is important in searches for Beyond Standard Model effects.

\section{References}

[1] M. Luszczak, W. Schäfer and A. Szczurek, "Two-photon dilepton production in proton-proton collisions: Two alternative approaches", Phys. Rev. D93 (2016) 074018.

[2] G. G. da Silveira, L. Forthomme, K. Piotrzkowski, W. Schäfer and A. Szczurek, "Central $\mu^{+} \mu$ production via photon-photon fusion in proton-proton collisions with proton dissociation," JHEP 1502, 159 (2015) [arXiv:1409.1541 [hep-ph]].

[3] A. Szczurek and G. Slipek, "Parton transverse momenta and Drell-Yan dilepton production," Phys. Rev. D 78 (2008) 114007 [arXiv:0808.1360 [hep-ph]]. 
[4] M. A. Nefedov, N. N. Nikolaev and V. A. Saleev, "Drell-Yan lepton pair production at high energies in the Parton Reggeization Approach,” Phys. Rev. D 87 (2013) 1, 014022 [arXiv:1211.5539 [hep-ph]].

[5] S. P. Baranov, A. V. Lipatov and N. P. Zotov, "Drell-Yan lepton pair production at the LHC and transverse momentum dependent quark densities of the proton,” Phys. Rev. D 89 (2014) 9, 094025 [arXiv:1402.5496 [hep-ph]].

[6] W. Schäfer and A. Szczurek, "Low mass Drell-Yan production of lepton pairs at forward directions at the LHC: A hybrid approach", Phys. Rev. D93 (2016) 074014.

[7] A. D. Martin, R. G. Roberts, W. J. Stirling and R. S. Thorne, "Parton distributions incorporating QED contributions," Eur. Phys. J. C 39, 155 (2005) [hep-ph/0411040].

[8] H. Abramowicz, E. M. Levin, A. Levy and U. Maor, “A Parametrization of sigma-T (gamma* p) above the resonance region Q**2 > = 0," Phys. Lett. B 269 (1991) 465.

[9] H. Abramowicz and A. Levy, "The ALLM parameterization of sigma(tot)(gamma* p): An Update," hep-ph/9712415.

[10] R. Fiore, A. Flachi, L. L. Jenkovszky, A. I. Lengyel and V. K. Magas, "Explicit model realizing parton hadron duality," Eur. Phys. J. A 15, 505 (2002) [hep-ph/0206027].

[11] M. M. Block, L. Durand and P. Ha, "Connection of the virtual $\gamma^{*} p$ cross section of ep deep inelastic scattering to real $\gamma p$ scattering, and the implications for $v N$ and $e p$ total cross sections," Phys. Rev. D 89, no. 9, 094027 (2014) [arXiv:1404.4530 [hep-ph]].

[12] A. Suri and D. R. Yennie, "The Space-time Phenomenology Of Photon Absorption And Inelastic Electron Scattering," Annals Phys. 72, 243 (1972).

[13] A. Szczurek and V. Uleshchenko, "Nonpartonic components in the nucleon structure functions at small Q**2 in the broad range of x,” Eur. Phys. J. C 12, 663 (2000) [hep-ph/9904288].

[14] J. Pumplin, D. R. Stump, J. Huston, H. L. Lai, P. M. Nadolsky and W. K. Tung, "New generation of parton distributions with uncertainties from global QCD analysis," JHEP 0207 (2002) 012 [hep-ph/0201195].

[15] A. Adare et al. [PHENIX Collaboration], "Dilepton mass spectra in $\mathrm{p}+\mathrm{p}$ collisions at $\mathrm{s}^{* *}(1 / 2)=$ 200-GeV and the contribution from open charm,", Phys. Lett. B 670 (2009) 313 [arXiv:0802.0050 [hep-ex]].

[16] [The LHCb collaboration], "Inclusive low mass Drell-Yan production in the forward region at sqrt(s)=7 TeV ," LHCb-CONF-2012-013; Conference report prepared for XX International Workshop on Deep-Inelastic Scattering and Related Subjects, 26-30, March 2012, Bonn, Germany.

[17] G. Aad et al. [ATLAS Collaboration], "Measurement of the low-mass Drell-Yan differential cross section at $\sqrt{s}=7 \mathrm{TeV}$ using the ATLAS detector," JHEP 1406, 112 (2014) [arXiv:1404.1212 [hep-ex]].

[18] G. Aad et al. [ATLAS Collaboration], "Measurement of the high-mass Drell-Yan differential cross-section in pp collisions at sqrt(s)=7 TeV with the ATLAS detector," Phys. Lett. B 725, 223 (2013) [arXiv:1305.4192 [hep-ex]].

[19] C. Kourkoumelis, L. K. Resvanis, T. A. Filippas, E. Fokitis, A. M. Cnops, J. H. Cobb, R. Hogue and S. Iwata et al., "Study of Massive Electron Pair Production at the CERN Intersecting Storage Rings," Phys. Lett. B 91 (1980) 475.

[20] S. Chatrchyan et al. [CMS Collaboration], "Search for exclusive or semi-exclusive photon pair production and observation of exclusive and semi-exclusive electron pair production in $p p$ collisions at $\sqrt{s}=7$ TeV," JHEP 1211, 080 (2012) [arXiv:1209.1666 [hep-ex]]. 